\title{
A Spatiotemporal Map of Reading Aloud
}

Oscar Woolnough ${ }^{1,2}$, Cristian Donos ${ }^{1,3}$, Aidan Curtis ${ }^{1}$, Patrick S. Rollo ${ }^{1,2}$, Zachary J. Roccaforte ${ }^{1,2}$, Stanislas Dehaene ${ }^{4,5}$, Simon Fischer-Baum ${ }^{6}$, Nitin Tandon ${ }^{1,2,7, *}$

${ }^{1}$ Vivian L. Smith Department of Neurosurgery, McGovern Medical School at UT Health Houston, Houston, TX, 77030, United States of America

${ }^{2}$ Texas Institute for Restorative Neurotechnologies, University of Texas Health Science Center at Houston, Houston, TX, 77030, United States of America

${ }^{3}$ Faculty of Physics, University of Bucharest, 050663, Bucharest, Romania

4 Cognitive Neuroimaging Unit CEA, INSERM, NeuroSpin Center, Université Paris-Sud and Université Paris-Saclay, 91191, Gif-sur-Yvette, France

${ }^{5}$ Collège de France, 11 Place Marcelin Berthelot, 75005, Paris, France

${ }^{6}$ Department of Psychological Sciences, Rice University, Houston, TX, 77005, USA

7 Memorial Hermann Hospital, Texas Medical Center, Houston, TX, 77030, United States of America

*Correspondence: nitin.tandon@uth.tmc.edu

\section{Abstract}

2 Reading words aloud is a foundational aspect of the acquisition of literacy. The rapid rate at which

3 multiple distributed neural substrates are engaged in this process can only be probed via 4 techniques with high spatiotemporal resolution. We used direct intracranial recordings in a large 5 cohort to create a holistic yet fine-grained map of word processing, enabling us to derive the spatiotemporal neural codes of multiple word attributes critical to reading: lexicality, word frequency and orthographic neighborhood. We found that lexicality is encoded by early activity in mid-fusiform (mFus) cortex and precentral sulcus. Word frequency is also first represented in mFus followed by 9 later engagement of the inferior frontal gyrus (IFG) and inferior parietal sulcus (IPS), and 10 orthographic neighborhood is encoded solely in the IPS. A lexicality decoder revealed high 11 weightings for electrodes in the mFus, IPS, anterior IFG and the pre-central sulcus. These results 12 elaborate the neural codes underpinning extant dual-route models of reading, with parallel 13 processing via the lexical route, progressing from mFus to IFG, and the sub-lexical route, 14 progressing from IPS to anterior IFG. 


\section{Introduction}

16 Reading a word aloud requires multiple complex transformations in the brain - mapping the visual

17 input of a letter string into an internal sequence of sound representations that are then expressed

18 through orofacial motor articulations. Models of how this mapping occurs during reading invoke a

19 dual-route architecture (Coltheart et al., 2001; Perry et al., 2007, 2010, 2019; Taylor et al., 2013),

20 with a lexico-semantic route for rapidly reading known words and a sub-lexical route for constructing

21 the phonology of novel words. A common method of targeting these two routes is to look at 22 contrasts between phonological exception words and pseudowords (Fiebach et al., 2002; Sebastian 23 et al., 2014; Shim et al., 2012; Taylor et al., 2013). Exception words contain irregular grapheme24 phoneme associations (e.g. yacht, sew) and their phonologies must be retrieved from internal 25 lexical representations as they cannot be accurately constructed de novo. In contrast, pseudowords 26 have no stored representation and their phonology must be constructed rather than retrieved.

27 Ventral temporal cortex, particularly mid-fusiform cortex (mFus), is strongly associated with the 28 lexical route. mFus is heavily implicated as the site of the orthographic lexicon, the long-term 29 memory storage of which letter strings map onto known words (Glezer et al., 2015; Hirshorn et al., 30 2016; Kronbichler et al., 2004; Lochy et al., 2018; White et al., 2019; Woolnough et al., 2021). This 31 region is sensitive to lexicality and word frequency (Kronbichler et al., 2004; White et al., 2019; 32 Woolnough et al., 2021), and shows selective changes during visual word learning (Glezer et al., 33 2015; Taylor et al., 2019). The sub-lexical route, essential for articulating novel words, is thought to 34 engage the inferior parietal lobe (IPL), dysfunction of which is associated with dyslexia (Raschle et 35 al., 2011; Temple et al., 2003; Tomasino et al., 2020), dysgraphia (Rapp et al., 2016), in addition to 36 phonological and semantic deficits (Binder et al., 2009; Hula et al., 2020; Numssen et al., 2021). 37 The two routes are proposed to converge in the inferior frontal gyrus (IFG) (Taylor et al., 2013).

38 The majority of our knowledge regarding the neural architecture underlying reading aloud is derived 39 from lesion data and functional MRI which provide accurate spatial localizations of function but lack 40 crucial temporal information. We utilized intracranial recordings in a large cohort of patients (44 41 patients, 3,642 electrodes), with medically intractable epilepsy, while they read aloud known and 42 novel words. This allowed us to comprehensively map the flow of information through these cortical 43 networks and track the spatiotemporal dynamics of the cortical representation of behaviorally 44 relevant lexical and sub-lexical factors.

\section{Results}

47 Participants were visually presented with phonologically regular words, exception words and novel 48 pseudowords that they read aloud (Figure 1A). Electrophysiological recordings were performed from 49 a total of 3,642 separate intracranial electrodes placed for the localization of intractable epilepsy 
(Figure 1B,C) - 4 participants had subdural grid electrodes (SDEs) and 40 had depth recordings using stereotactic EEG electrodes (sEEGs).
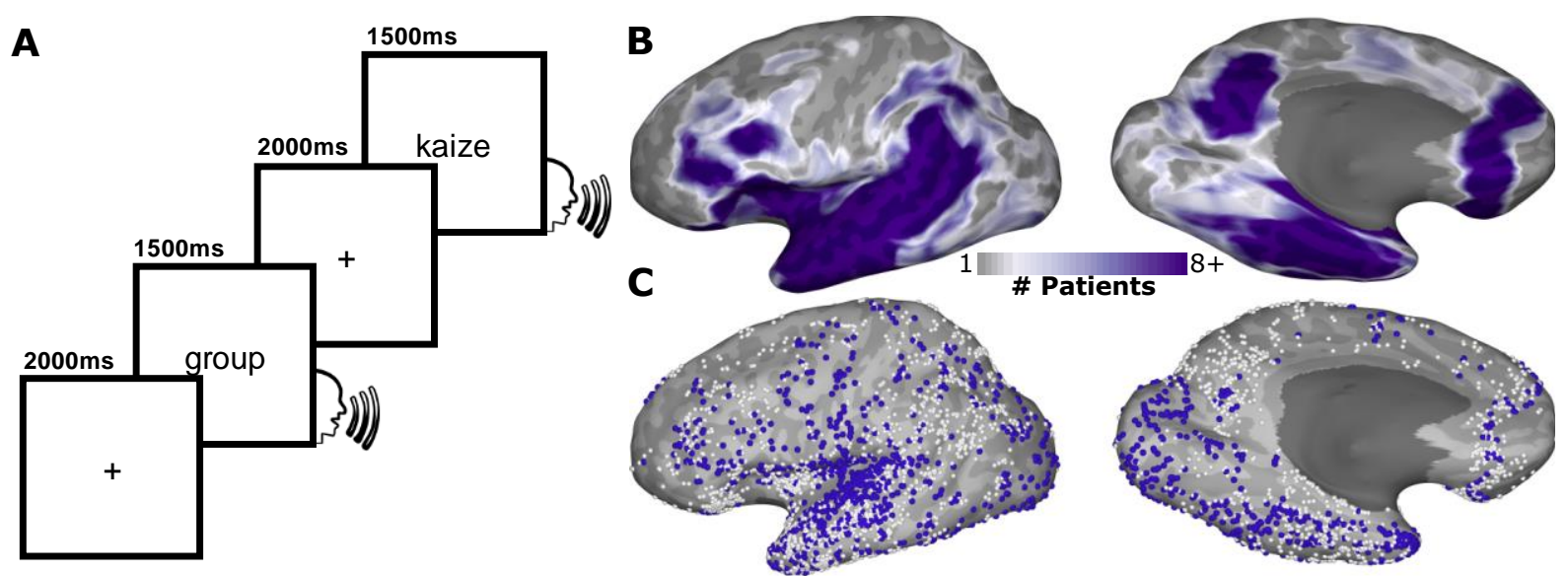

Figure 1: Experimental Design and Electrode Coverage. (A) Schematic representation of the reading task. (B) Representative coverage map (44 patients) and (C) individual electrode locations $(3,642$ electrodes) for the left hemisphere, highlighting responsive electrodes (1,158 electrodes; $>20 \%$ activation above baseline).

\section{Behavioral Analysis}

59 Mean ( $\pm \mathrm{SD}$ ) response times (RTs) were: regular words (743 $\pm 122 \mathrm{~ms})$, exception words $(747 \pm$ $60125 \mathrm{~ms}$ ) and pseudowords (923 $\pm 193 \mathrm{~ms}$ ) (Figure 2A). Regular and exception words showed no difference in RT (Wilcoxon sign rank, $p=0.75$; $\ln \left(\right.$ Bayes Factor $\left.\left(B_{10}\right)\right)=-1.5$ ) though pseudoword RT was slower than for exception words $\left(p<10^{-8}, \ln \left(B F_{10}\right)=28\right)$.
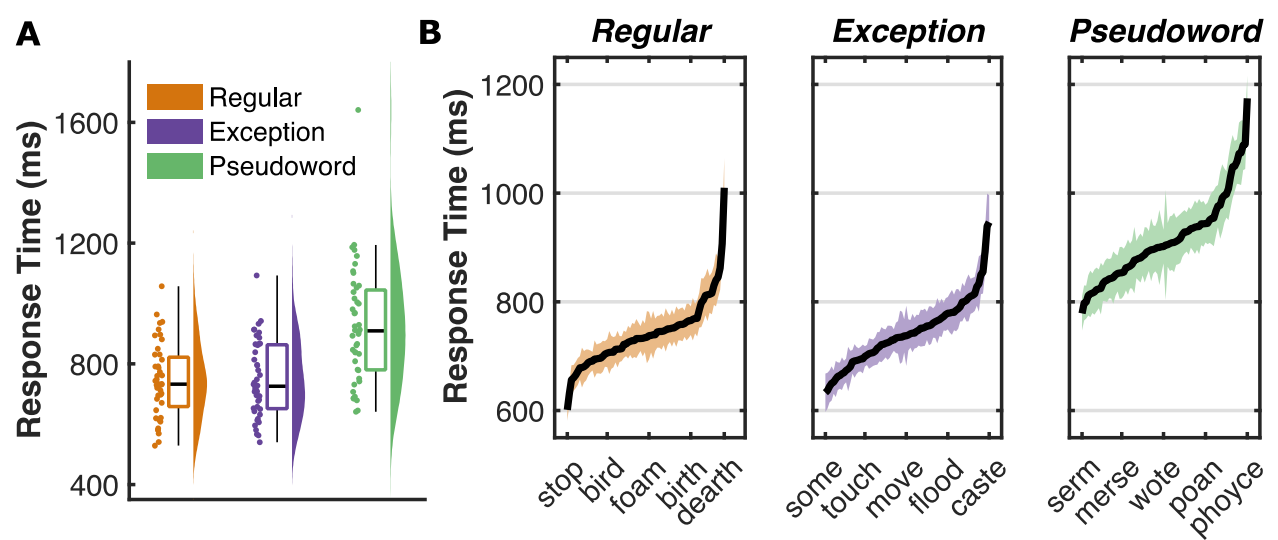

64 Figure 2: Population Word Response Times. (A) Response time distribution for each of the three word classes, averaged within participant, $(B)$ Mean ( \pm SE) response times for each item within the three word classes, averaged across participants.

68 To determine the underlying properties of the words that modulate RT within this cohort, we performed linear mixed effects (LME) and Bayes factor (BF) analyses on each word class with fixed 
70 effects modelling linguistic factors commonly linked to word identification and articulation (Table 1).

71 Regular words and exception word RTs showed the greatest modulation by word frequency.

72 Pseudoword RT was most strongly associated with orthographic neighborhood.

\begin{tabular}{|c|c|c|c|c|c|c|c|c|c|}
\hline & \multicolumn{3}{|c|}{$\begin{array}{c}\text { Regular } \\
\mathrm{df}=3170, \mathrm{r}^{2}=0.36\end{array}$} & \multicolumn{3}{|c|}{$\begin{array}{c}\text { Exception } \\
\mathrm{df}=3098, r^{2}=0.35\end{array}$} & \multicolumn{3}{|c|}{$\begin{array}{c}\text { Pseudowords } \\
\mathrm{df}=3185, \mathrm{r}^{2}=0.40\end{array}$} \\
\hline & $\beta$ (SE) & $\mathbf{p}$ & $\ln \left(B F_{10}\right)$ & $\beta$ (SE) & $\mathbf{p}$ & $\ln \left(B_{10}\right)$ & $\beta$ (SE) & $\mathbf{p}$ & $\ln \left(B_{10}\right)$ \\
\hline Length & $\begin{array}{c}49 \\
(17) \\
\end{array}$ & 0.004 & 0.9 & $\begin{array}{c}48 \\
(22) \\
\end{array}$ & 0.03 & -0.7 & $\begin{array}{c}23 \\
(26) \\
\end{array}$ & 0.38 & -3 \\
\hline Word Frequency & $\begin{array}{l}-186 \\
(17) \\
\end{array}$ & $<10^{-27}$ & 59 & $\begin{array}{l}-154 \\
(16) \\
\end{array}$ & $<10^{-21}$ & 43 & - & - & - \\
\hline $\begin{array}{l}\text { Orthographic } \\
\text { Neighborhood }\end{array}$ & $\begin{array}{c}52 \\
(27)\end{array}$ & 0.05 & -0.9 & $\begin{array}{l}-97 \\
(35)\end{array}$ & 0.005 & 1.4 & $\begin{array}{l}227 \\
(33)\end{array}$ & $<10^{-11}$ & 21 \\
\hline $\begin{array}{l}\text { Phonological } \\
\text { Neighborhood }\end{array}$ & $\begin{array}{c}20 \\
(18)\end{array}$ & 0.26 & -2.5 & $\begin{array}{c}-7 \\
(16)\end{array}$ & 0.63 & -3.2 & $\begin{array}{c}58 \\
(20)\end{array}$ & 0.004 & 0.5 \\
\hline $\begin{array}{l}\text { Positional Letter } \\
\text { Frequency }\end{array}$ & $\begin{array}{c}13 \\
(14)\end{array}$ & 0.89 & -2.9 & $\begin{array}{l}-16 \\
(16)\end{array}$ & 0.29 & -2.9 & $\begin{array}{l}-50 \\
(19)\end{array}$ & 0.009 & -0.3 \\
\hline
\end{tabular}

74 Table 1: Statistical Modelling of Response Time. As predictors were normalized, $\beta$ values

75 approximate change in RT between extreme values within the entire stimulus set (Supplementary

76 Table 1). Factors with strong evidence of an effect $\left(\ln \left(\mathrm{BF}_{10}\right)>2.3\right)$ are highlighted.

\section{Spatiotemporal Mapping of Single Word Reading}

79 We used a mixed-effects, multilevel analysis (MEMA) of broadband gamma activity (BGA; 70-150

$80 \mathrm{~Hz}$ ) in group surface normalized space to create a population level map of cortical activation across

81 the population. This analysis is specifically designed to account for sampling variations and to

82 minimize effects of outliers (Argall et al., 2006; Conner et al., 2014; Esposito et al., 2013; Fischl et

83 al., 1999; Kadipasaoglu et al., 2014; Saad and Reynolds, 2012). All correctly articulated trials

84 across all word classes, were used. 4D representations of the spread of activation across the

85 cortical surface were generated by performing MEMA on short, overlapping time windows (150 ms

86 width, $10 \mathrm{~ms}$ spacing) to generate successive images of cortical activity, time locked to stimulus

87 onset (Video 1) or the onset of articulation (Video 2). The spatial distribution of activations was

88 highly comparable across word classes (Supplementary Figure 1).

89 By collapsing across these frames, we visualized peak activations at each point on the cortical

90 surface (Figure $3 \mathrm{~A}$ ). To create a more focused visualization of the spatiotemporal progression

91 across reading-sensitive cortex, we selected 12 regions of interest (ROIs) in areas thought to be

92 important to written word processing, speech production and speech monitoring (Figure 3B,C). This

93 analysis highlights regions displaying primarily pre-articulatory processes, in ventral

94 occipitotemporal cortex, inferior parietal lobe and the inferior frontal gyrus. 

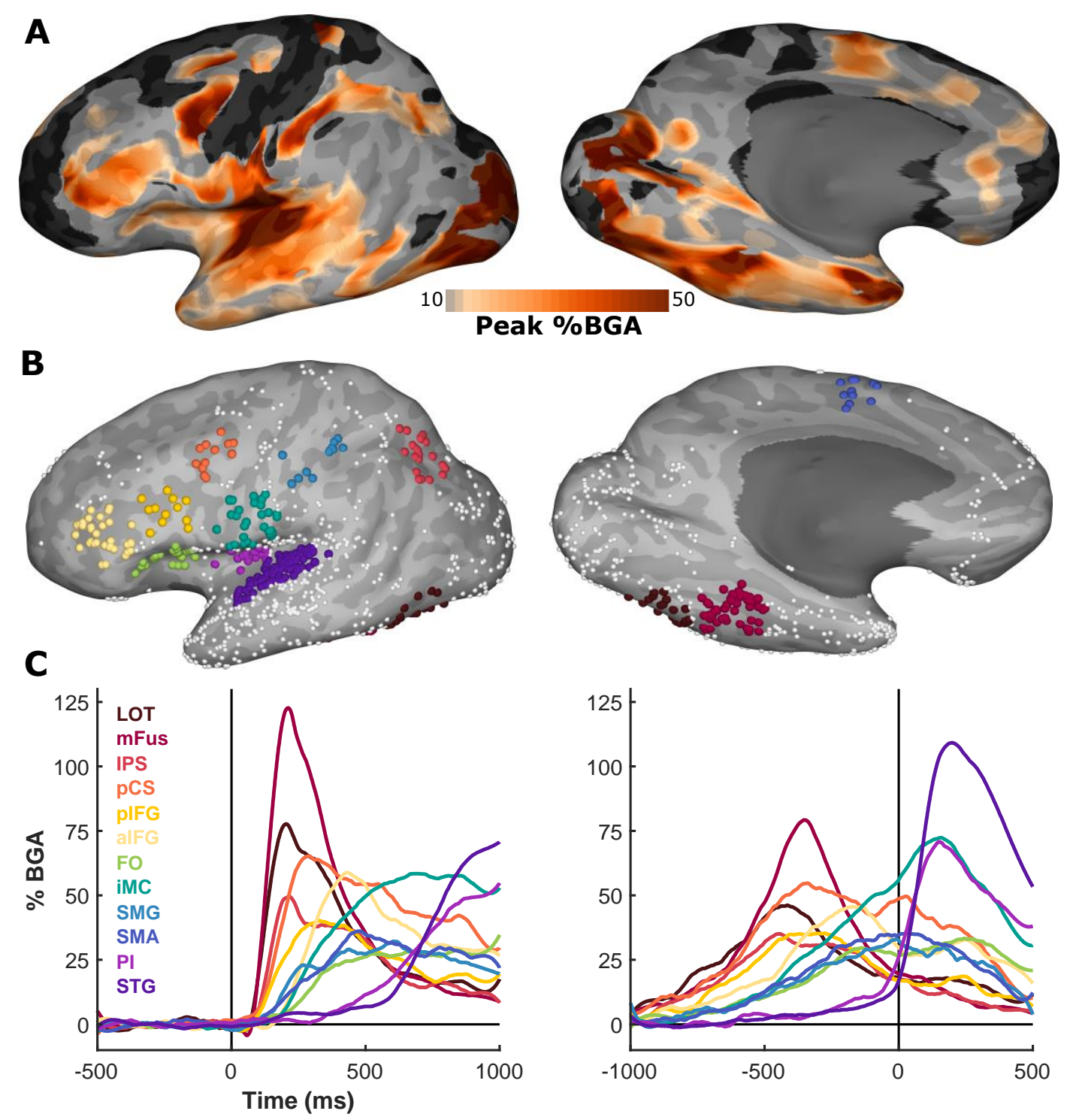

96 Figure 3: Spatiotemporal Profile of Cortical Activations. (A) Collapsed articulation-locked activation movie (Video 2) highlighting the amplitude of peak activation. (B) Representative ROls in 12 anatomically and functionally distinct regions, showing all responsive electrodes. (C) Mean activation during word reading of each $\mathrm{ROI}$, averaged within patient, time locked to stimulus onset 100 (left) and articulation onset (right). Standard errors omitted for visual clarity. LOT, Lateral 101 OccipitoTemporal cortex; mFus, mid-Fusiform Cortex; IPS, Inferior Parietal Sulcus; pCS, pre102 Central Sulcus; pIFG, posterior Inferior Frontal Gyrus; alFG, anterior Inferior Frontal Gyrus; FO, 103 Frontal Operculum; iMC, inferior Motor Cortex; SMG, Supra Marginal Gyrus; SMA, Supplementary 104 Motor Area; PI, Posterior Insula; STG, Superior Temporal Gyrus.

\section{Spatiotemporal Representation of Lexical Factors}

107 To distinguish activity patterns across word classes we contrasted grouped gamma power 108 activations between exception vs. pseudowords (lexicality) and exception vs. regular words 109 (regularity) using MEMA. The lexicality contrasts demonstrated clusters in mFus, precentral sulcus 110 (pCS), inferior parietal sulcus (IPS) and anterior inferior frontal gyrus (alFG). 
111 To determine how distinguishable whole-network activity patterns are for each of these factors,

112 within-individuals at a single trial level, we used a logistic regression decoder. Decoders trained to

113 distinguish between exception word and pseudoword trials demonstrated high decoding accuracy,

114 with some patients showing $>80 \%$ decoding accuracy (Figure 4C). These lexicality decoders

115 displayed high electrode weightings across the ventral temporal surface, IPS, pCS and alFG (Figure

116 4D). Decoders trained to distinguish exception and regular words did not show higher decoding 117 accuracy than in the baseline period.
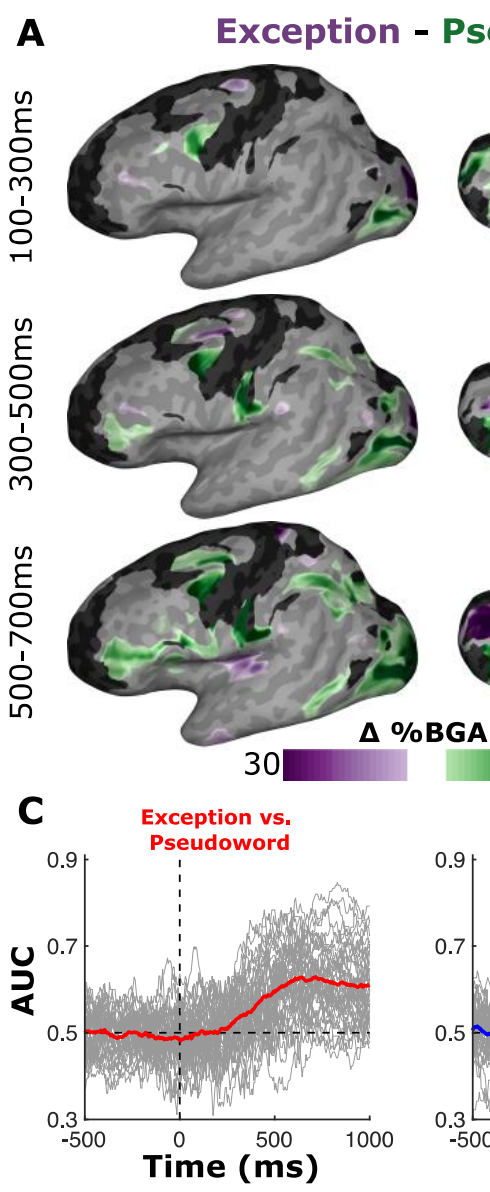
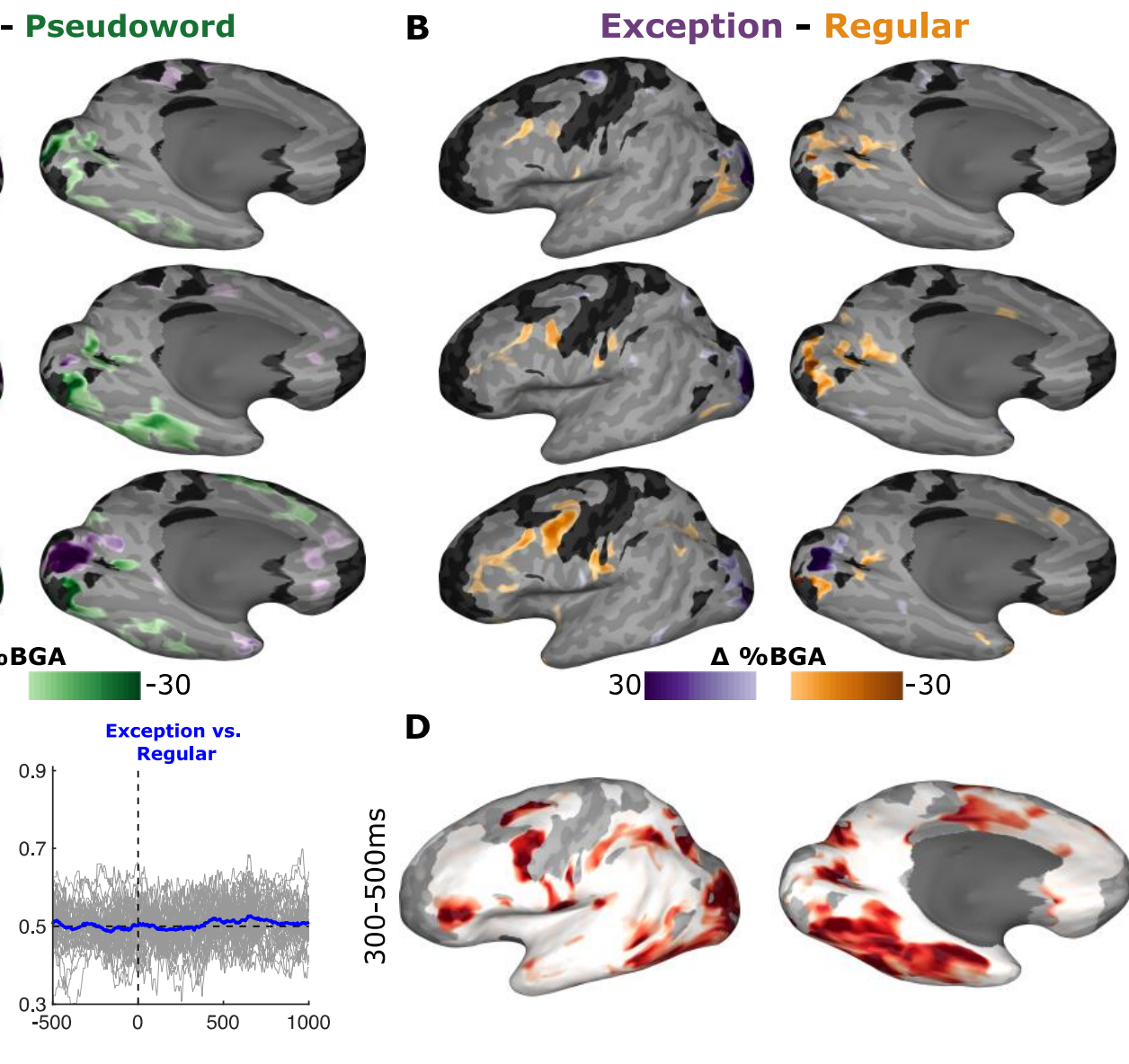

D
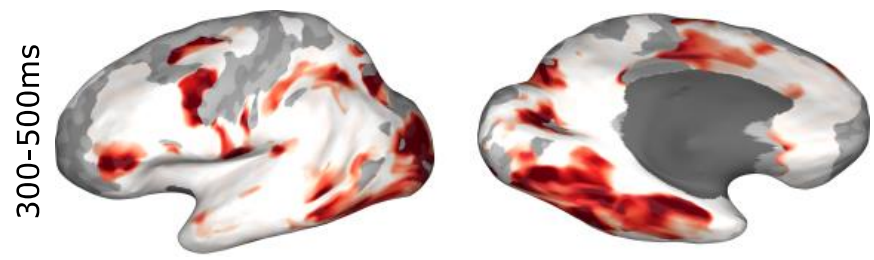

119 Figure 4: Contrasting Word Classes. (A,B) MEMA contrasts of $(A)$ exception - pseudoword and

120 (B) exception - regular, revealing regions of significantly different BGA between conditions $(p<0.01$

121 corrected). Regions in black did not have consistent coverage for reliable MEMA results. (C)

122 Decoding accuracies of the logistic regression decoders trained to distinguish exception word vs.

123 pseudoword trials (left) and exception word vs regular word trials (right). Grey lines represent

124 individual patient decoding accuracies. Colored line represents median accuracy. (D) Cortical

125 surface representation of population average electrode weightings of the exception vs pseudoword

126 decoder between $300-500$ ms.

128 We observed lexicality distinctions between known words (regular and exception) and novel 129 pseudowords broadly across the previously defined ROls (Figure 5). These distinctions were 130 observed earliest in mFus before spreading to pCS and visual word form regions, and subsequently 
to IFG and IPS. Distinctions were also observed in post-articulatory auditory regions (posterior

132 insula and superior temporal gyrus) relating to differences in RT between known and novel words.
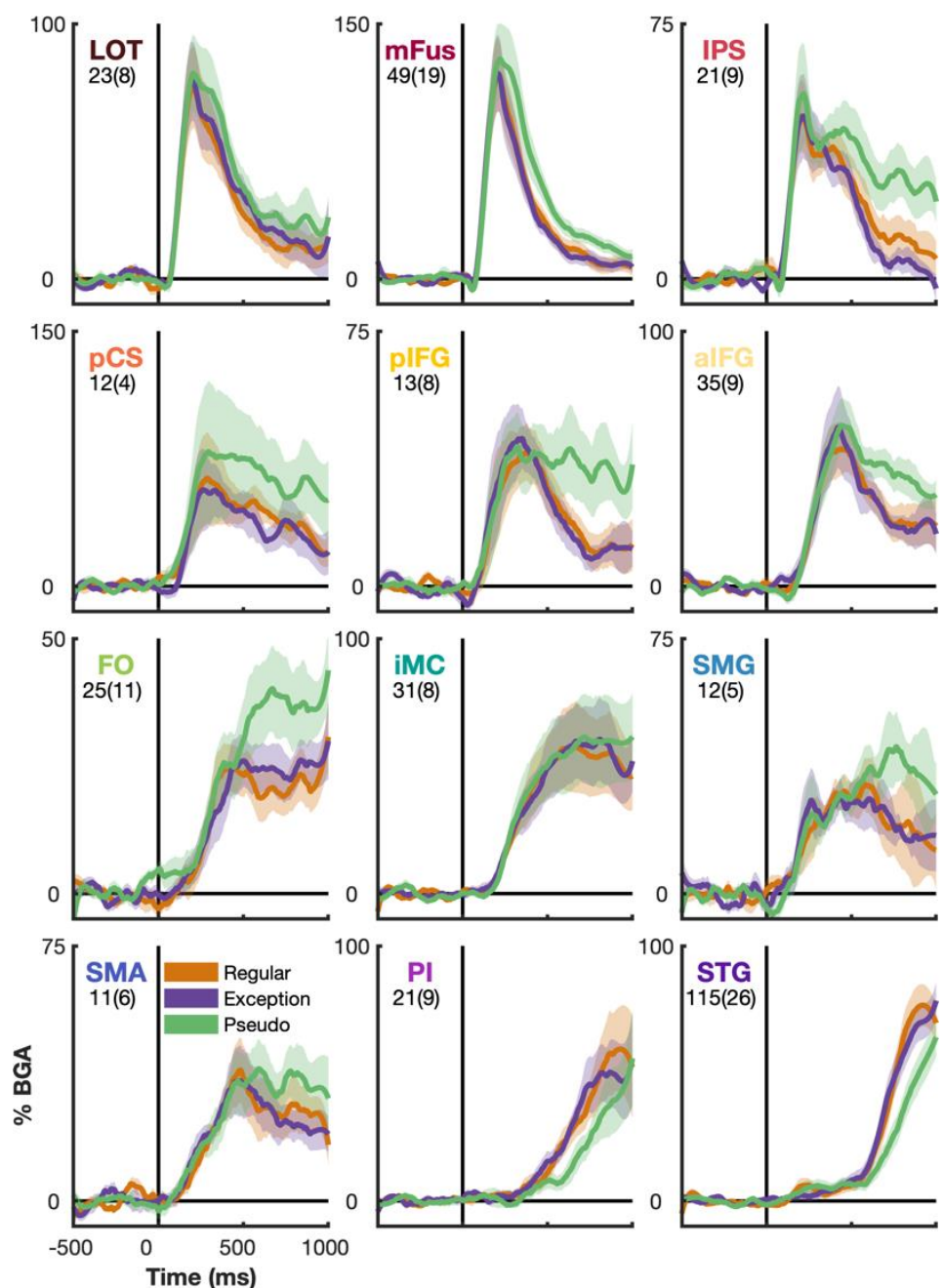

134 Figure 5: Spatiotemporal Activation Profiles of Known and Novel Words. Mean activation $( \pm$ $\mathrm{SE}$ ) for each word class, within each ROI, during word reading, averaged within patient, time locked

136 to stimulus onset. Number of electrodes and patients, per ROI, is indicated. LOT, Lateral 137 OccipitoTemporal cortex; mFus, mid-Fusiform Cortex; IPS, Inferior Parietal Sulcus; pCS, pre138 Central Sulcus; pIFG, posterior Inferior Frontal Gyrus; alFG, anterior Inferior Frontal Gyrus; FO, 139 Frontal Operculum; iMC, inferior Motor Cortex; SMG, Supra Marginal Gyrus; SMA, Supplementary 140 Motor Area; PI, Posterior Insula; STG, Superior Temporal Gyrus.

142 For the six ROls that showed a clear pre-articulatory peak in activation, we analyzed their activity for 143 sensitivity to the main drivers of RT seen in the behavioral analysis; lexicality, word frequency of 144 known words and orthographic neighborhood of pseudowords. mFus showed the earliest sensitivity 145 to lexicality, followed by LOT and pCS, and then broad sensitivity across multiple regions (Figure 146 6A). mFus showed an early and long-lasting word frequency sensitivity, with IPS and alFG becoming sensitive later (500-700 ms). Sensitivity to orthographic neighborhood of pseudowords was only seen in IPS $(500-700 \mathrm{~ms})$. 
149 For the three regions we found to have evidence of word frequency or orthographic neighborhood

150 sensitivity, mFus, IPS and alFG, we used LME models at a higher time resolution. Sensitivity to

151 word frequency was observed earliest in mFus (200 ms) followed by IPS and alFG (425 ms) (Figure

152 6B). In IPS we observed a period of elevated orthographic neighborhood sensitivity, but this did not

153 show significance at this time resolution (Figure 6C).

A

\begin{tabular}{r|c|c|c|}
\multicolumn{3}{c}{ Lexicality } \\
\cline { 2 - 4 } LOT & -1.7 & 7.3 & 1.4 \\
\cline { 2 - 4 } mFus & 4.6 & 92 & $>100$ \\
IPS & -4.3 & -1.2 & 26 \\
\cline { 2 - 4 } pCS & -3.1 & 2.4 & 4.2 \\
\cline { 2 - 4 } pIFG & -3.5 & -4.2 & 18 \\
\cline { 2 - 4 } AIFG & -4.2 & -2.4 & 18 \\
\cline { 2 - 4 } & &
\end{tabular}

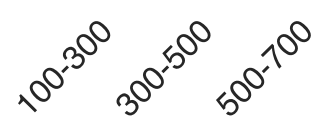

Time (ms)

B

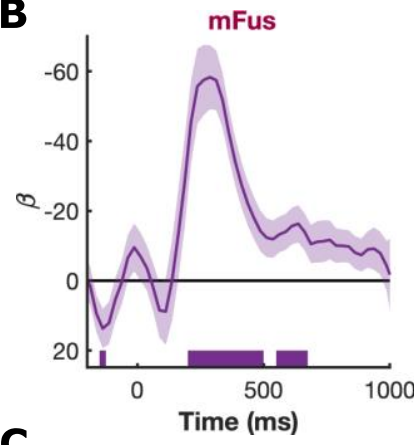

c

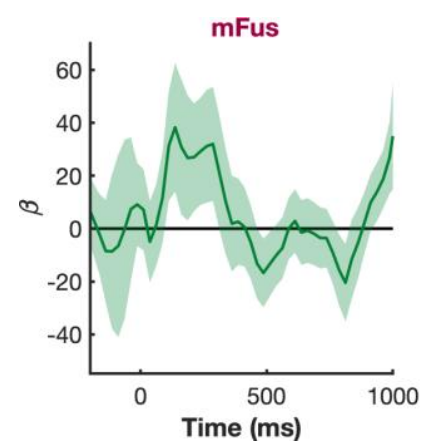

Word

Frequency

\begin{tabular}{|c|c|c|}
\hline-2 & -2.2 & -2.6 \\
\hline 4.3 & 16 & 3 \\
\hline-2.7 & -0.97 & 5.3 \\
\hline-2 & -1.6 & -2 \\
\hline-2.9 & -2.8 & 0.19 \\
\hline-3 & 2.3 & 13 \\
\hline
\end{tabular}

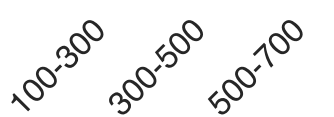

IPS
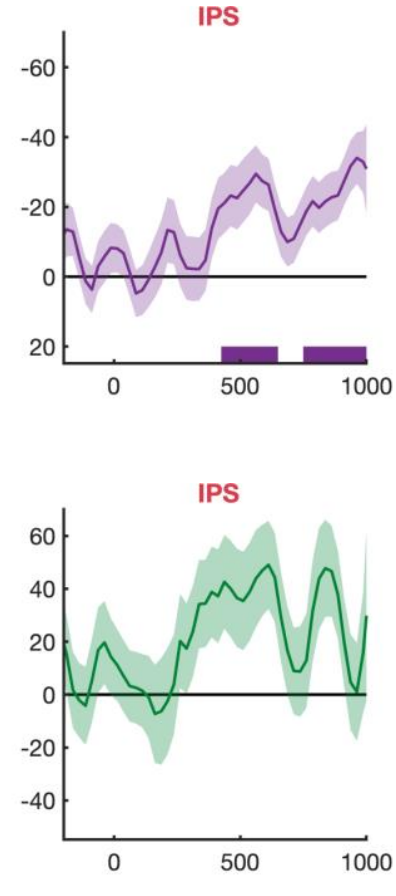

Orthographic

Neighborhood

\begin{tabular}{|c|c|c|}
\hline-1.6 & -1 & -2.4 \\
\hline-0.038 & -2.9 & -3.3 \\
\hline-2.2 & 2.2 & 4.8 \\
\hline-0.77 & -1.7 & -1.6 \\
\hline-0.68 & -2 & -1.7 \\
\hline-2.2 & -2.8 & -2.8 \\
\hline
\end{tabular}
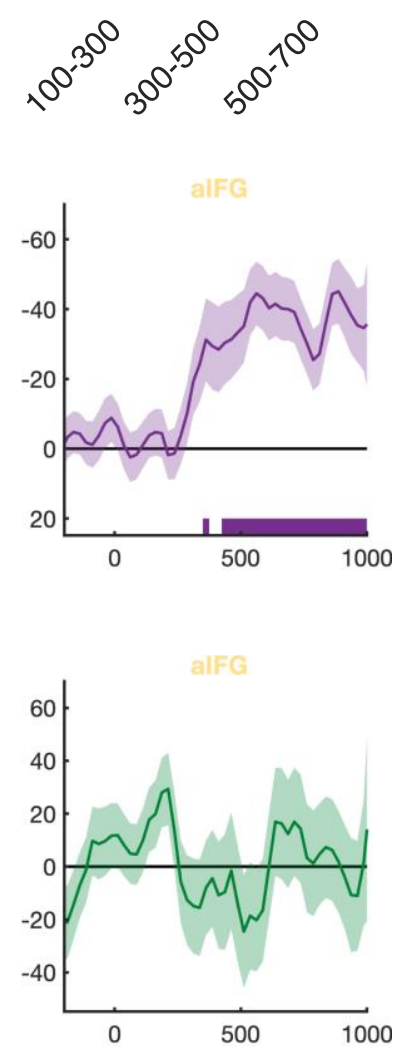

155 Figure 6: Regression of Lexical Factors. (A) Bayes factor analysis of lexicality, word frequency 156 and orthographic neighborhood effects in the six pre-articulatory ROls, for three time windows. 157 Lexicality tested all known words against pseudowords. Word frequency was regressed across all 158 known words. Orthographic neighborhood was regressed across all pseudowords. Bayes factor $159\left(\ln \left(B F_{10}\right)\right)$ shown for each contrast and values $>2.3$ are highlighted. $(B, C)$ Linear mixed effects 160 (LME) model regression of (B) word frequency in known words and (C) orthographic neighborhood 161 in pseudowords, in three ROls ( $\beta \pm$ SE; mFus, 49 electrodes, 19 patients; IPS, 21 electrodes, 9 162 patients; alFG, 35 electrodes, 9 patients). Colored bars represent regions of significance $(q<0.05)$. 


\section{Discussion}

165 This large population intracranial study comprehensively maps the spatiotemporal spread of cortical 166 activation across the left hemisphere during word reading to derive the dynamics of cortical 167 networks underlying literacy. Dual-route architectures of reading networks (Coltheart et al., 2001; 168 Perry et al., 2007, 2010, 2019), derived on behavioral and lesional data, imply separable processing 169 routes for known vs novel words. We find a network of regions sensitive to lexicality, initially the 170 mFus and pCS, then spreading broadly across IPS and IFG. The spatial distribution of this lexicality 171 effect is broadly comparable with the lexicality network identified with fMRI (Heim et al., 2013; Taylor 172 et al., 2013, 2014), with the added benefit of millisecond temporal resolution. Responses in lexicality 173 sensitive regions maximally separate for known and novel words between 300-500ms after stimulus 174 onset, in a manner that is reliable enough to enable single trial decoding of lexicality. These data 175 minimize the impact of response time variations, which confounds modalities with lower temporal 176 resolution (e.g. fMRI) and may artificially inflate lexicality effects in regions such as IFG (Taylor et 177 al., 2014).

178 We have previously demonstrated that mFus is the earliest region in ventral temporal cortex to show 179 sensitivity to word frequency while reading (Woolnough et al., 2021). It is commonly assumed that 180 sensitivity to statistical properties of language such as word frequency seen in ventral temporal 181 cortex are as a result of top-down modulation from IFG (Heim et al., 2013; Price and Devlin, 2011; 182 Woodhead et al., 2014). Here, we demonstrate again the primacy of the mFus in coding both word 183 frequency and lexicality, preceding the engagement of aIFG and IPS in these processes by over $184200 \mathrm{~ms}$. This consolidates mFus's role as a specialized orthographic lexicon, organized based on 185 statistical regularities of individual words in natural language.

186 The IPS was the only region with sensitivity to orthographic neighborhood. This sensitivity likely 187 reflects grapheme-phoneme conversion processes in this region (Dehaene-Lambertz et al., 2018; 188 Xu et al., 2020). Given that IPS shows both word frequency and lexicality sensitivity, its role in sub189 lexical processing might appear to be questionable. However, for known words, the lexical route is 190 faster and more accurate than the sub-lexical route - thus, once a letter string is identified as a 191 known lexical object, sub-lexical processes are no longer required. Given the word frequency 192 dependence of lexical identification, the timing of the cessation of sub-lexical processes should also 193 be frequency dependent. This interpretation is entirely consistent with our data as IPS shows more 194 sustained activity, but not higher peak activity for novel words.

195 It is theorized that pCS is involved in articulatory phonological processing, specifically feedforward 196 control of articulator velocity (Matchin and Hickok, 2020; Tourville and Guenther, 2011). Through 197 lesion studies pCS has also been linked to phonological dyslexia (Rapcsak et al., 2009; Tomasino 198 et al., 2020). Our data demonstrate that pCS activation begins early, preceding the IFG, suggesting 199 a role in early linguistic or phonological processing, potentially as part of the sub-lexical route. pCS 200 demonstrates lexical sensitivity but no effect of word frequency. Given the association of pCS with 
201 articulation phonology and phonological dyslexia, this may represent part of the process of 202 constructing novel phonologies.

203 This study provides further evidence that medial frontal operculum is involved in pre-articulatory, 204 preparatory processes, distinct from those of the lateral IFG (Mălîia et al., 2018; Woolnough et al., 205 2019). Lesions involving this region have been linked to impairment of complex articulation (Baldo 206 et al., 2011) which may explain the greater engagement during pseudoword articulation.

207 We observed no significant pre-articulatory activity in middle temporal gyrus or angular gyrus, 208 regions that have been linked to semantic and phonological processes during word processing 209 (Graves et al., 2010; Hartwigsen et al., 2010; Sliwinska et al., 2015; Stoeckel et al., 2009). These 210 regions appear to be engaged during reading in children but may not be recruited in adults for 211 simple reading tasks (Martin et al., 2015), instead being used primarily for comprehending multi212 word phrases (Dronkers et al., 2004; Fridriksson et al., 2018; Matchin et al., 2017).

\section{Materials and Methods}

215 Participants: 44 patients (25 male, 19-60 years, 5 left-handed, IQ $94 \pm$ 15, Age of Epilepsy Onset 18 $216 \pm 9$ years) participated in the experiments after giving written informed consent. All participants were 217 semi-chronically implanted with intracranial electrodes for seizure localization of pharmaco-resistant 218 epilepsy. Participants were excluded if they had confirmed right-hemisphere language dominance or 219 a significant additional neurological history (e.g. previous resections, MR imaging abnormalities 220 such as malformations or hypoplasia). All experimental procedures were reviewed and approved by 221 the Committee for the Protection of Human Subjects (CPHS) of the University of Texas Health 222 Science Center at Houston as Protocol Number HSC-MS-06-0385.

223 Electrode Implantation and Data Recording: Data were acquired from either subdural grid 224 electrodes (SDEs; 4 patients) or stereotactically placed depth electrodes (sEEGs; 40 225 patients). SDEs were subdural platinum-iridium electrodes embedded in a silicone elastomer sheet 226 (PMT Corporation; top-hat design; 3mm diameter cortical contact), and were surgically implanted 227 via a craniotomy (Pieters et al., 2013; Tandon, 2012; Tong et al., 2020). sEEGs were implanted 228 using a Robotic Surgical Assistant (ROSA; Medtech, Montpellier, France) (Rollo et al., 2020; 229 Tandon et al., 2019). Each sEEG probe (PMT corporation, Chanhassen, Minnesota) was $0.8 \mathrm{~mm}$ in 230 diameter and had 8-16 electrode contacts. Each contact was a platinum-iridium cylinder, $2.0 \mathrm{~mm}$ in 231 length and separated from the adjacent contact by $1.5-2.43 \mathrm{~mm}$. Each patient had 12-20 such 232 probes implanted. Following surgical implantation, electrodes were localized by co-registration of 233 pre-operative anatomical 3T MRI and post-operative CT scans in AFNI (Cox, 1996). Electrode 234 positions were projected onto a cortical surface model generated in FreeSurfer (Dale et al., 1999), 235 and displayed on the cortical surface model for visualization (Pieters et al., 2013). Intracranial data 236 were collected during research experiments starting on the first day after electrode implantation for 
237 sEEGs and two days after implantation for SDEs. Data were digitized at $2 \mathrm{kHz}$ using the NeuroPort 238 recording system (Blackrock Microsystems, Salt Lake City, Utah), imported into Matlab, initially 239 referenced to the white matter channel used as a reference for the clinical acquisition system and 240 visually inspected for line noise, artifacts and epileptic activity. Electrodes with excessive line noise 241 or localized to sites of seizure onset were excluded. Each electrode was re-referenced to the 242 common average of the remaining channels. Trials contaminated by inter-ictal epileptic spikes were 243 discarded.

244 Stimuli and Experimental Design: All patients undertook a word reading task. Stimuli were 245 presented on a 2,880 x 1,800 pixel, 15.4" LCD screen positioned at eye-level, 2-3' from the patient.

246 Participants were presented with 80 each of monosyllabic (i) phonologically regular words, (ii) 247 phonologically irregular exception words and (iii) novel pseudowords and asked to read them aloud.

248 Stimuli were presented using Psychophysics Toolbox (Kleiner et al., 2007) in Matlab, in all lower249 case letters, in Arial font with a height of 150 pixels $\left(\sim 2.2^{\circ}\right.$ visual angle). Each stimulus was 250 displayed for $1,500 \mathrm{~ms}$ with an inter-stimulus interval of 2,000 ms. Stimuli were presented in two 251 recording sessions, each containing presentation of 120 stimuli in a pseudorandom order with no 252 repeats. $95 \pm 4 \%$ of trials were correctly articulated. The most common errors were regularization of 253 exception words (e.g. sew as sue, soot as sute) or lexicalization of pseudowords (e.g. shret as 254 shirt, jinje as jingle).

255 Signal Analysis: Analyses were performed by first bandpass filtering raw data of each electrode into 256 broadband gamma activity $(B G A ; 70-150 \mathrm{~Hz}$ ) following removal of line noise (zero-phase 2nd order 257 Butterworth bandstop filters). A frequency domain bandpass Hilbert transform (paired sigmoid flanks 258 with half-width $1.5 \mathrm{~Hz}$ ) was applied and the analytic amplitude was smoothed (Savitzky - Golay finite 259 impulse response, 3rd order, frame length of $201 \mathrm{~ms}$ ). BGA is presented here as percentage 260 change from baseline level, defined as the period -500 to $-100 \mathrm{~ms}$ before each word presentation.

261 Electrodes were tested to see if they were responsive during the task. Responsiveness was defined 262 as displaying $>20 \%$ average BGA over baseline for at least one of the three following windows: 100 263 to $500 \mathrm{~ms}$ following stimulus onset, -500 to $-100 \mathrm{~ms}$ before articulation onset or 100 to $500 \mathrm{~ms}$ 264 following articulation onset. Of the 3,642 useable electrodes, 1,158 electrodes were designated 265 responsive based on these criteria.

266 Neural Decoding: Decoding analyses were performed using logistic regression classifiers, using 5267 fold cross validation, implemented within MNE-Python (Gramfort, 2013; Gramfort et al., 2014). For 268 each patient, decoding performance was summarized with an area under the curve (AUC) and a set 269 of classifier weights for each electrode. Temporal decoding was performed on BGA using a sliding 270 estimator at each time point, using all available electrodes. Spatial distribution of classifier weights 271 was reconstructed by a cortical surface transform onto a standardized brain surface using each 272 electrode's presumed "recording zone", an exponentially decaying geodesic radius (Kadipasaoglu et 
273 al., 2014). Cortical surface maps were amplitude normalized within patient then averaged across

274 patient to create a population weighting map.

275 Linguistic Analysis: We quantified word frequency as the base-10 log of the SUBTLEXus frequency

276 (Brysbaert and New, 2009). This resulted in a frequency of 1 meaning 10 instances per million

277 words and 4 meaning 10,000 instances per million words. There was no significant difference

278 between word frequency of regular (1.5 \pm 0.35 ; Mean \pm SD) and exception (1.7 \pm 1.0$)$ words

279 (Wilcoxon rank sum, $p=0.36$ ). Positional letter frequency was calculated as the base-10 log of the

280 sum of the SUBTLEXus frequencies of all words with a given letter in a specific ordinal position.

281 Orthographic neighborhood was quantified as the orthographic Levenshtein distance (OLD20); the

282 mean number of single character edits required to convert the word into its 20 nearest neighbors

283 with a log frequency greater than 0 (Yarkoni et al., 2008). Phonological neighborhood densities

284 were obtained from the Irvine Phonotactic Online Dictionary (IPhOD) (Vaden et al., 2009).

285 Pseudowords were phonemically transcribed using the most common pronunciation. 


\section{Acknowledgements}

287 We express our gratitude to all the patients who participated in this study; the neurologists at the 288 Texas Comprehensive Epilepsy Program who participated in the care of these patients; and the 289 nurses and technicians in the Epilepsy Monitoring Unit at Memorial Hermann Hospital who helped 290 make this research possible. This work was supported by the National Institute of Neurological 291 Disorders and Stroke NS098981.

\section{Author Contributions}

293 Conceptualization: OW, CD, SD, NT; Methodology: OW, CD, NT; Data curation: OW, CD, PSR, ZR; 294 Software: OW, CD, AC; Formal Analysis: OW, AC; Writing - Original Draft: OW; Writing - Review 295 and Editing: OW, SD, SFB, NT; Visualization: OW; Funding Acquisition: NT; Neurosurgical 296 Procedures: NT.

\section{Declaration of Interests}

298 The authors declare no competing interests 


\section{References}

301 Argall, B.D., Saad, Z.S., and Beauchamp, M.S. (2006). Simplified intersubject averaging on the cortical surface using SUMA. Hum. Brain Mapp. 27, 14-27.

303 Baldo, J. V, Wilkins, D.P., Ogar, J., Willock, S., and Dronkers, N.F. (2011). Role of the precentral 304 gyrus of the insula in complex articulation. Cortex 47, 800-807.

305 Binder, J.R., Desai, R.H., Graves, W.W., and Conant, L.L. (2009). Where is the semantic system? A 306 critical review and meta-analysis of 120 functional neuroimaging studies. Cereb. Cortex 19, 27673072796.

308 Brysbaert, M., and New, B. (2009). Moving beyond Kučera and Francis: A critical evaluation of current word frequency norms and the introduction of a new and improved word frequency measure for American English. Behav. Res. Methods 41, 977-990.

Coltheart, M., Rastle, K., Perry, C., Langdon, R., and Ziegler, J. (2001). DRC: A dual route

313 Conner, C.R., Chen, G., Pieters, T.A., and Tandon, N. (2014). Category specific spatial

314 dissociations of parallel processes underlying visual naming. Cereb. Cortex 24, 2741-2750.

315 Cox, R.W. (1996). AFNI: Software for Analysis and Visualization of Functional Magnetic Resonance 316 Neuroimages. Comput. Biomed. Res. 29, 162-173.

317 Dale, A.M., Fischl, B., and Sereno, M.I. (1999). Cortical Surface-Based Analysis: I. Segmentation 318 and Surface Reconstruction. Neuroimage 9, 179-194.

319 Dehaene-Lambertz, G., Monzalvo, K., and Dehaene, S. (2018). The emergence of the visual word 320 form: Longitudinal evolution of category-specific ventral visual areas during reading acquisition. 321 PLOS Biol. 16, e2004103.

322 Dronkers, N.F., Wilkins, D.P., Van Valin, R.D., Redfern, B.B., and Jaeger, J.J. (2004). Lesion 323 analysis of the brain areas involved in language comprehension. Cognition 92, 145-177.

324 Esposito, F., Singer, N., Podlipsky, I., Fried, I., Hendler, T., and Goebel, R. (2013). Cortex-based 325 inter-subject analysis of $\mathrm{EEEG}$ and fMRI data sets: Application to sustained task-related BOLD and 326 gamma responses. Neuroimage 66, 457-468.

327 Fiebach, C.J., Friederici, A.D., Müller, K., and Von Cramon, D.Y. (2002). fMRI evidence for dual 328 routes to the mental lexicon in visual word recognition. J. Cogn. Neurosci. 14, 11-23.

329 Fischl, B., Sereno, M.I., Tootell, R.B.H., and Dale, A. (1999). High-resolution inter-subject averaging 330 and a surface-based coordinate system. Hum. Brain Mapp. 8, 272-284.

331 Fridriksson, J., Den Ouden, D.B., Hillis, A.E., Hickok, G., Rorden, C., Basilakos, A., Yourganov, G., 332 and Bonilha, L. (2018). Anatomy of aphasia revisited. Brain 141, 848-862.

333 Glezer, L.S., Kim, J., Rule, J., Jiang, X., and Riesenhuber, M. (2015). Adding Words to the Brain's 
334 Visual Dictionary: Novel Word Learning Selectively Sharpens Orthographic Representations in the

335 VWFA. J. Neurosci. 35, 4965-4972.

336 Gramfort, A. (2013). MEG and EEG data analysis with MNE-Python. Front. Neurosci. 7, 1-13.

337 Gramfort, A., Luessi, M., Larson, E., Engemann, D.A., Strohmeier, D., Brodbeck, C., Parkkonen, L., 338 and Hämäläinen, M.S. (2014). MNE software for processing MEG and EEG data. Neuroimage 86, $339446-460$.

340 Graves, W.W., Desai, R., Humphries, C., Seidenberg, M.S., and Binder, J.R. (2010). Neural 341 systems for reading aloud: A multiparametric approach. Cereb. Cortex 20, 1799-1815.

342 Hartwigsen, G., Baumgaertner, A., Price, C.J., Koehnke, M., Ulmer, S., and Siebner, H.R. (2010).

343 Phonological decisions require both the left and right supramarginal gyri. Proc. Natl. Acad. Sci. U. S. 344 A. $107,16494-16499$.

345 Heim, S., Wehnelt, A., Grande, M., Huber, W., and Amunts, K. (2013). Effects of lexicality and word 346 frequency on brain activation in dyslexic readers. Brain Lang. 125, 194-202.

347 Hirshorn, E.A., Li, Y., Ward, M.J., Richardson, R.M., Fiez, J.A., and Ghuman, A.S. (2016). Decoding 348 and disrupting left midfusiform gyrus activity during word reading. Proc. Natl. Acad. Sci. 113, 81623498167.

350 Hula, W.D., Panesar, S., Gravier, M.L., Yeh, F.-C., Dresang, H.C., Dickey, M.W., and Fernandez351 Miranda, J.C. (2020). Structural white matter connectometry of word production in aphasia: an 352 observational study. Brain 143, 2532-2544.

353 Kadipasaoglu, C.M., Baboyan, V.G., Conner, C.R., Chen, G., Saad, Z.S., and Tandon, N. (2014).

354 Surface-based mixed effects multilevel analysis of grouped human electrocorticography.

355 Neuroimage 101, 215-224.

356 Kleiner, M., Brainard, D., and Pelli, D. (2007). What's new in Psychtoolbox-3? Perception 36.

357 Kronbichler, M., Hutzler, F., Wimmer, H., Mair, A., Staffen, W., and Ladurner, G. (2004). The visual 358 word form area and the frequency with which words are encountered: Evidence from a parametric 359 fMRI study. Neuroimage 21, 946-953.

360 Lochy, A., Jacques, C., Maillard, L., Colnat-Coulbois, S., Rossion, B., and Jonas, J. (2018).

361 Selective visual representation of letters and words in the left ventral occipito-temporal cortex with 362 intracerebral recordings. Proc. Natl. Acad. Sci. 115, E7595-E7604.

363 Mălîia, M.-D., Donos, C., Barborica, A., Popa, I., Ciurea, J., Cinatti, S., and Mîndru, I. (2018). 364 Functional mapping and effective connectivity of the human operculum. Cortex 109, 303-321.

365 Martin, A., Schurz, M., Kronbichler, M., and Richlan, F. (2015). Reading in the brain of children and 366 adults: A meta-analysis of 40 functional magnetic resonance imaging studies. Hum. Brain Mapp. 36, 367 1963-1981. 
Matchin, W., and Hickok, G. (2020). The Cortical Organization of Syntax. Cereb. Cortex 30, 14811498.

Matchin, W., Hammerly, C., and Lau, E. (2017). The role of the IFG and pSTS in syntactic prediction: Evidence from a parametric study of hierarchical structure in fMRI. Cortex 88, 106-123.

Numssen, O., Bzdok, D., and Hartwigsen, G. (2021). Functional specialization within the inferior parietal lobes across cognitive domains. Elife 10.

Perry, C., Ziegler, J.C., and Zorzi, M. (2007). Nested incremental modeling in the development of computational theories: The CDP+ model of reading aloud. Psychol. Rev. 114, 273-315.

Perry, C., Ziegler, J.C., and Zorzi, M. (2010). Beyond single syllables: Large-scale modeling of reading aloud with the Connectionist Dual Process (CDP++) model. Cogn. Psychol. 61, 106-151.

Perry, C., Zorzi, M., and Ziegler, J.C. (2019). Understanding Dyslexia Through Personalized LargeScale Computational Models. Psychol. Sci. 30, 386-395.

Pieters, T.A., Conner, C.R., and Tandon, N. (2013). Recursive grid partitioning on a cortical surface model: an optimized technique for the localization of implanted subdural electrodes. J. Neurosurg. 118, 1086-1097.

Price, C.J., and Devlin, J.T. (2011). The Interactive Account of ventral occipitotemporal contributions to reading. Trends Cogn. Sci. 15, 246-253.

Rapcsak, S.Z., Beeson, P.M., Henry, M.L., Leyden, A., Kim, E., Rising, K., Andersen, S., and Cho, H.S. (2009). Phonological dyslexia and dysgraphia: Cognitive mechanisms and neural substrates. Cortex 45, 575-591.

Rapp, B., Purcell, J., Hillis, A.E., Capasso, R., and Miceli, G. (2016). Neural bases of orthographic long-term memory and working memory in dysgraphia. Brain 139, 588-604.

Raschle, N.M., Chang, M., and Gaab, N. (2011). Structural brain alterations associated with dyslexia predate reading onset. Neuroimage $57,742-749$.

Rollo, P.S., Rollo, M.J., Zhu, P., Woolnough, O., and Tandon, N. (2020). Oblique trajectory angles in robotic stereo-electroencephalography. J. Neurosurg.

Saad, Z.S., and Reynolds, R.C. (2012). Suma. Neuroimage 62, 768-773. occipitotemporal cortex in reading, spelling, and naming. Cogn. Neuropsychol. 31, 511-528. neuropsychological correlates of spelling errors in primary progressive aphasia. Neuropsychologia

400 Sliwinska, M.W., James, A., and Devlin, J.T. (2015). Inferior Parietal Lobule Contributions to Visual 401 Word Recognition. J. Cogn. Neurosci. 27, 593-604. 
402

403

404

405

406

407

408

409

410

411

412

413

Stoeckel, C., Gough, P.M., Watkins, K.E., and Devlin, J.T. (2009). Supramarginal gyrus involvement in visual word recognition. Cortex 45, 1091-1096.

Tandon, N. (2012). Mapping of human language. In Clinical Brain Mapping, D. Yoshor, and E. Mizrahi, eds. (McGraw Hill Education), pp. 203-218.

Tandon, N., Tong, B.A., Friedman, E.R., Johnson, J.A., Von Allmen, G., Thomas, M.S., Hope, O.A., Kalamangalam, G.P., Slater, J.D., and Thompson, S.A. (2019). Analysis of Morbidity and Outcomes Associated With Use of Subdural Grids vs Stereoelectroencephalography in Patients With Intractable Epilepsy. JAMA Neurol. 76, 672-681.

Taylor, J.S.H., Rastle, K., and Davis, M.H. (2013). Can cognitive models explain brain activation during word and pseudoword reading? A meta-analysis of 36 neuroimaging studies. Psychol. Bull. 139, 766-791.

Taylor, J.S.H., Rastle, K., and Davis, M.H. (2014). Interpreting response time effects in functional imaging studies. Neuroimage 99, 419-433.

Taylor, J.S.H., Davis, M.H., and Rastle, K. (2019). Mapping visual symbols onto spoken language along the ventral visual stream. Proc. Natl. Acad. Sci. 116, 17723-17728.

Temple, E., Deutsch, G.K., Poldrack, R.A., Miller, S.L., Tallal, P., Merzenich, M.M., and Gabrieli, J.D.E. (2003). Neural deficits in children with dyslexia ameliorated by behavioral remediation:

Evidence from functional MRI. Proc. Natl. Acad. Sci. U. S. A. 100, 2860-2865.

Tomasino, B., lus, T., Skrap, M., and Luzzatti, C. (2020). Phonological and surface dyslexia in individuals with brain tumors: Performance pre-, intra-, immediately post-surgery and at follow-up. Hum. Brain Mapp. 41, 5015-5031.

Tong, B.A., Esquenazi, Y., Johnson, J., Zhu, P., and Tandon, N. (2020). The Brain is Not Flat: Conformal Electrode Arrays Diminish Complications of Subdural Electrode Implantation, A Series of 117 Cases. World Neurosurg. 144, e734-e742.

Tourville, J.A., and Guenther, F.H. (2011). The DIVA model: A neural theory of speech acquisition and production. Lang. Cogn. Process. 26, 952-981.

Vaden, K.I., Halpin, H.R., and Hickok, G.S. (2009). Irvine Phonotactic Online Dictionary, Version 2.0. [Data file].

White, A.L., Palmer, J., Boynton, G.M., and Yeatman, J.D. (2019). Parallel spatial channels converge at a bottleneck in anterior word-selective cortex. Proc. Natl. Acad. Sci. 116, 10087-10096.

Woodhead, Z.V.J., Barnes, G.R., Penny, W., Moran, R., Teki, S., Price, C.J., and Leff, A.P. (2014). Reading front to back: MEG evidence for early feedback effects during word recognition. Cereb. Cortex 24, 817-825.

Woolnough, O., Forseth, K.J., Rollo, P.S., and Tandon, N. (2019). Uncovering the functional 
436 anatomy of the human insula during speech. Elife 8 , e53086.

437 Woolnough, O., Donos, C., Rollo, P.S., Forseth, K.J., Lakretz, Y., Crone, N.E., Fischer-Baum, S., 438 Dehaene, S., and Tandon, N. (2021). Spatiotemporal dynamics of orthographic and lexical 439 processing in the ventral visual pathway. Nat. Hum. Behav. 5, 389-398.

440 Xu, W., Kolozsvari, O.B., Oostenveld, R., and Hämäläinen, J.A. (2020). Rapid changes in brain 441 activity during learning of grapheme-phoneme associations in adults. Neuroimage 220, 117058.

442 Yarkoni, T., Balota, D., and Yap, M. (2008). Moving beyond Coltheart's N: A new measure of 443 orthographic similarity. Psychon. Bull. Rev. 15, 971-979. 


\section{Supplementary Information}

446 Video 1: Spread of Stimulus-Locked Activity across the Cortical Surface. MEMA movie of the 447 time course of broadband gamma activation across the cortical surface with trials time-locked to 448 onset of the visual stimulus. Regions in black did not have consistent coverage for reliable MEMA 449 results.

451 Video 2: Spread of Articulation-Locked Activity across the Cortical Surface. MEMA movie of 452 the time course of broadband gamma activation across the cortical surface with trials time locked to 453 the onset of articulation. Regions in black did not have consistent coverage for reliable MEMA 454 results.

\begin{tabular}{|c|c|c|c|c|c|c|c|c|c|c|c|c|}
\hline & \multicolumn{3}{|c|}{ All } & \multicolumn{3}{|c|}{ Regular } & \multicolumn{3}{|c|}{ Exception } & \multicolumn{3}{|c|}{ Pseudowords } \\
\hline & Min & Med & Max & Min & Med & Max & Min & Med & Max & Min & Med & Max \\
\hline Length & 3 & 4 & 6 & 3 & 4 & 6 & 3 & 4 & 6 & 4 & 4 & 6 \\
\hline Word Frequency & -1 & 1.7 & 3.8 & -1 & 1.7 & 3 & -0.5 & 1.8 & 3.8 & - & - & - \\
\hline $\begin{array}{l}\text { Orthographic } \\
\text { Neighborhood }\end{array}$ & 1 & 1.7 & 2.8 & 1 & 1.8 & 2.4 & 1 & 1.6 & 2 & 1.2 & 1.9 & 2.8 \\
\hline $\begin{array}{l}\text { Phonological } \\
\text { Neighborhood }\end{array}$ & 0 & 22 & 49 & 6 & 24 & 42 & 1 & 22 & 49 & 0 & 19 & 41 \\
\hline $\begin{array}{l}\text { Positional Letter } \\
\text { Frequency }\end{array}$ & 4.4 & 4.9 & 5.2 & 4.4 & 4.9 & 5.1 & 4.5 & 4.9 & 5.1 & 4.5 & 4.8 & 5.2 \\
\hline
\end{tabular}

457 values for each of the regressors used, across the whole stimulus set and for individual word

458 classes. Statistical models used normalized data, subtracting the minimum value and dividing by the range across the whole stimulus set. 


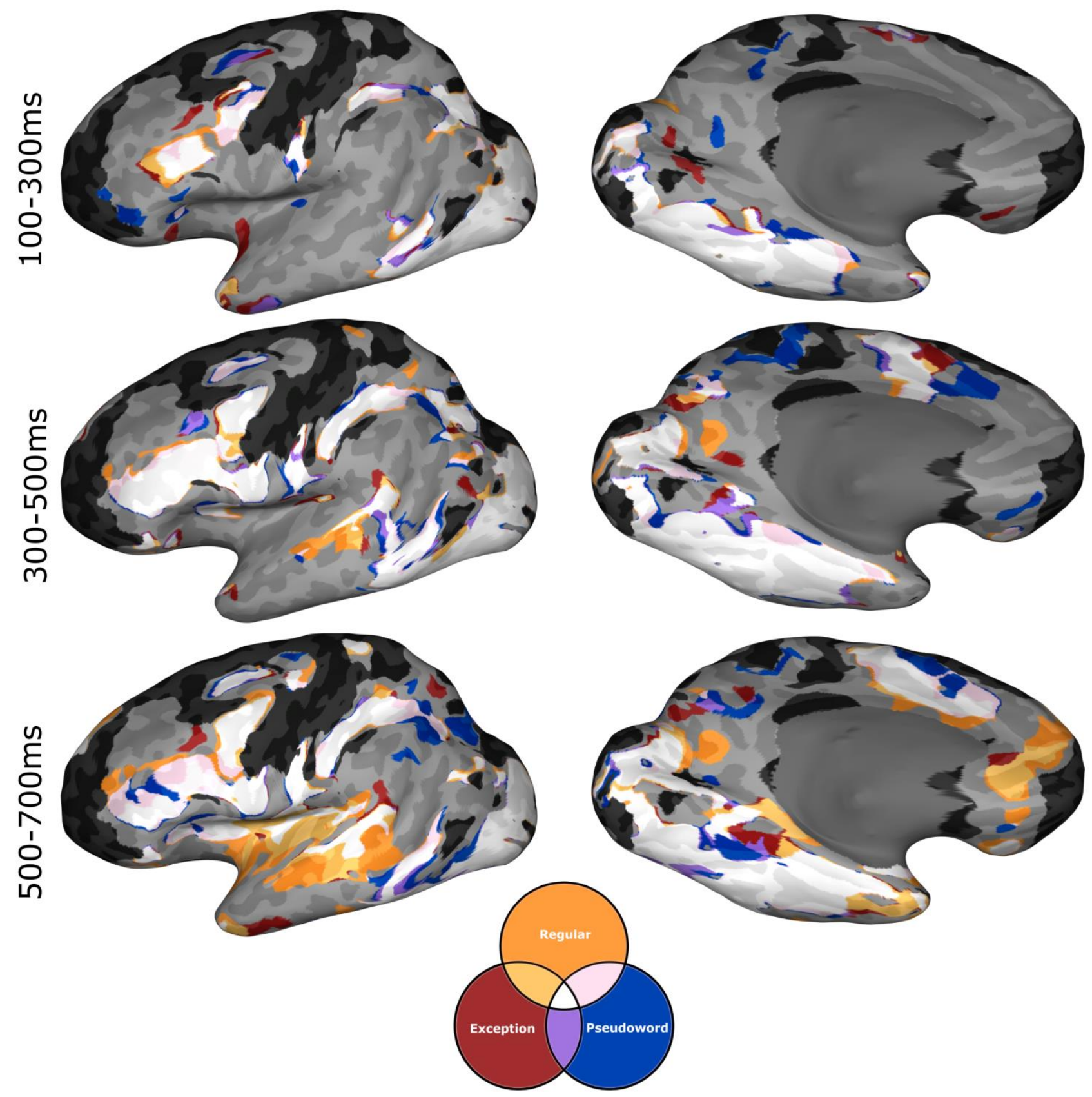

462 Supplementary Figure 1: Conjunction Map of Word Class Activations. MEMA conjunction maps

463 showing overlap of binarized activation maps of each of the three word classes tested (\%BGA >

$4645 \%, t>2.58$, patients $\geq 3$ ), over three time windows locked to stimulus onset. Across all time

465 windows all three word classes demonstrate a gross overlap of activation (white). In the later time

466 window, areas associated with post-articulatory processes (e.g. auditory cortex) show selective

467 activation for known words, reflecting differences in response time between known words and novel

468 pseudowords. Regions in black did not have consistent coverage for reliable MEMA results. 